\title{
PI3K/Akt signaling pathway is involved in the neurotrophic effect of senegenin
}

\author{
TING PI ${ }^{1,2}$, XIAO-WEN ZHOU ${ }^{3}$, LIANG CAI $^{2}$, WEI ZHANG ${ }^{2}, \mathrm{CHAO-FEN} \mathrm{SU}^{2}$, \\ WU-TIAN WU ${ }^{4}$, XIAO-MING REN ${ }^{1}$ and HUAN-MIN LUO ${ }^{2,5}$
}

\author{
${ }^{1}$ Department of Pharmacy, Yan'an Hospital Affiliated to Kunming Medical University, Kunming, Yunnan 650051; \\ ${ }^{2}$ Department of Pharmacology, School of Medicine, Jinan University, Guangzhou, Guangdong 510632; \\ ${ }^{3}$ Department of Pharmacy, Guangzhou Chest Hospital, Guangzhou, Guangdong 510095; ${ }^{4}$ Department of Anatomy, \\ Li Ka-shing Faculty of Medicine of The University of Hong Kong, Hong Kong 999077, SAR; ${ }^{5}$ Department of \\ Pharmacology, Institute of Brain Sciences, Jinan University, Guangzhou, Guangdong 510632, P.R. China
}

Received December 25, 2014; Accepted September 18, 2015

DOI: $10.3892 / \mathrm{mmr} .2015 .4652$

\begin{abstract}
Neurodegenerative diseases are frequently associated with the loss of synapses and neurons. Senegenin, extracted from the Chinese herb Polygala tenuifolia Willd, was previously found to promote neurite outgrowth and neuronal survival in primary cultured rat cortical neurons. The aim of the present study was to investigate the underlying mechanisms of senegenin-induced neurotrophic effects on rat cortical neurons. Primary cortical rat neurons were treated with various pharmacological antagonists and with or without senegenin, and subjected to MTT and western blot analysis to explore the effects of senegenin on cell survival as well as the activation of signaling pathways. Neurite outgrowth and neuronal survival induced by senegenin were significantly inhibited by $\mathrm{A}_{2 \mathrm{~A}}$ receptor antagonist ZM241385 and specific phosphoinositide-3 kinase (PI3K) inhibitor LY294002, but not by tropomyosin receptor kinase A receptor inhibitor K252a, mitogen-activated protein kinase kinase inhibitor PD98059 or protein kinase C inhibitor GÖ6976. Furthermore, senegenin enhanced the phosphorylation of Akt, which was blocked by LY294002. The present study revealed that the PI3K/Akt signaling pathway may be involved in the neurotrophic effects of senegenin.
\end{abstract}

Correspondence to: Professor Huan-Min Luo, Department of Pharmacology, School of Medicine, Jinan University, 601 Huangpu Avenue West, Guangzhou, Guangdong 510632, P.R. China E-mail: tlhm@jnu.edu.cn

Abbreviations: DMEM/F12, Dulbecco's modified Eagle's medium: nutrient mixture F12; MAP2, microtubule-asscociated protein 2; MTT, 3-(4,5-dimethylthiazol-2-yl)-2,5-diphenyltetrazolium bromide; NTF, neurotrophic factor

Key words: neurotrophic effects, neurotrophic factors, senegenin, phosphoinositide-3 kinase, neurodegenerative diseases

\section{Introduction}

Excessive neuronal cell loss through synapse loss and neurite damage is a common characteristic of numerous brain diseases, particularly of neurodegenerative diseases, including Alzheimer's, Parkinson's and Huntington's disease (1). Studies have shown that several endogenous neurotrophic factors (NTFs), including brain-derived neurotrophic factor (BDNF), nerve growth factor (NGF) as well as neurotrophin-3, -4 and -5 have critical roles in neuronal survival, process outgrowth, synaptic connectivity and nervous system plasticity $(2,3)$. Thus, NTFs are likely to have a potential role in ameliorating neurodegeneration. However, due to their peptidyl properties and their resulting inability to cross the blood-brain barrier as well as their fast metabolic breakdown, the clinical use of NTFs is impossible (4). Identification of small molecules with similar functions to those of NTFs may provide novel therapeutics for neurodegenerative diseases. Of note, numerous compounds, including 4-O-methylhonokiol, magnolol and macranthol, have been identified to promote neurite process outgrowth and neuronal survival due to their similar functions to those of NTFs in vitro (5-8).

Senegenin is a compound extracted from the Chinese herb Polygala tenuifolia Willd and has been shown to exert a range of biological activities, including neuroprotective and antioxidant effects $(9,10)$. A pharmacological study showed that Polygala tenuifolia Willd extracts improve cognitive function and have neuroprotective properties (11). Previous studies by our group revealed that senegenin promoted neurite outgrowth and upregulated the mRNA expression of microtubule-associated protein 2 (MAP2) and brain-derived neurotrophic factor in cultured cortical neurons $(12,13)$. The present study further investigated the neurotrophic effects of senegenin as well as the underlying mechanisms.

\section{Materials and methods}

Materials. Senegenin was purchased from Guangdong Institute for Drug Control (Guangzhou, China). Dulbecco's modified Eagle's medium (DMEM)/F12 and B27 supplement 
were from Gibco (Thermo Fisher Scientific, Waltham, MA, USA). K252a, GÖ6976, PD98059, LY294002 and MAP2 antibody were purchased from Sigma-Aldrich (St. Louis, MO, USA). ZM241385 was purchased from Tocris Bioscience Co. Ltd. (Bristol, UK). Paraformaldehyde was purchased from Guangzhou Chemical Reagent Factory (Guangzhou, China). Trypsin was purchased from Amresco, LLC (Solon, OH, USA). The cell strainer was obtained from Dingguo Biotechnology Co., Ltd. (Beijing, China). Gibco goat serum was purchased from Thermo Fisher Scientific, Inc. The Hoechst stain, lysis buffer components, and electrochemiluminescence reagent (ECL; cat no. P0018) were purchased from Beyotime Institute of Biotechnology (Shanghai, China). Polyvinylidene fluoride (PVDF) membranes and bicinchoninic acid (BCA) assay were purchased from Sigma-Aldrich. The anti-MAP2 mouse monoclonal antibody (cat no. M9942) was purchased from Sigma-Aldrich. Anti-Akt (pan) rabbit monoclonal antibody (cat no. C67E7) and anti-phospho-Akt monoclonal antibody (Ser473) rabbit monoclonal antibody (cat no. 4060) were purchased from Cell Signaling Technology, Inc. (Danvers, MA, USA). Cy3 Affini Pure goat anti-mouse polyclonal IgG (H+L; (cat no. 115-095-003) was purchased from Jackson ImmunoResearch Laboratories, Inc. (West Grove, PA, USA). Rabbit GAPDH polyclonal antibody (cat no. 10494-1-AP) was purchased from Proteintech Group, Inc. (Chicago, IL, USA). Horseradish peroxidase (HRP)-conjugated goat anti-rabbit IgG secondary antibody $(\mathrm{H}+\mathrm{L}$; cat no. A0208) was purchased from Beyotime Institute of Biotechnology, Inc.

Primary culture of cortical neurons. Neonatal Sprague-Dawley (SD) rats $(<24 \mathrm{~h}$ after birth) obtained from the Medical Laboratory Animal Center of Guangdong province [license no. CXK (Yue) 2008-0002] were used for the preparation of the primary culture of cortical neurons as described previously (14). Briefly, after neonatal SD rats were decapitated under anesthesia (diethyl ether; Beyotime Institute of Biotechnology), the brains were placed into petri dishes containing ice-cold D-Hank's balanced salt solution. Cortices were separated and minced into small pieces. The cells were dissociated by digestion with $0.125 \%(\mathrm{w} / \mathrm{v})$ trypsin for $10 \mathrm{~min}$ at $37^{\circ} \mathrm{C}$ followed by passing through a $74-\mu \mathrm{m}$ cell strainer and centrifugation for $5 \mathrm{~min}$ at $1,000 \mathrm{x} \mathrm{g}$. The cell pellet was re-suspended to the desired concentration with DMEM/F12 supplemented with $0.4 \%$ (v/v) B27. Freshly isolated cells were cultured in poly-L-lysine-coated 24-/96-well plates. These cells were cultured at $37^{\circ} \mathrm{C}$ in a humidified atmosphere containing $5 \% \mathrm{CO}_{2}$. After three days of culture, cells were collected for subsequent experiments. The study was approved by the ethics committee of the Medical School of Jinan University (Guangzhou, China).

Immunocytochemistry assay. Cortical neurons were identified by immunostaining for MAP2, which is a specific neuronal marker. After 3 days of culture, cells were fixed with $4 \%$ $(\mathrm{w} / \mathrm{v})$ paraformaldehyde at room temperature for $20 \mathrm{~min}$ and subsequently incubated with $0.1 \%(\mathrm{v} / \mathrm{v})$ Triton X-100 in phosphate-buffered saline (PBS) containing $10 \%(\mathrm{v} / \mathrm{v})$ normal goat serum for $30 \mathrm{~min}$. Cells were then incubated with mouse anti-MAP2 monoclonal antibody (1:200 dilution) overnight at $4^{\circ} \mathrm{C}$ followed by staining with $\mathrm{Cy} 3 \mathrm{AffiniPure}$ goat anti-mouse
IgG (1:500 dilution) for $1 \mathrm{~h}$ and Hoechst 33258 for $5 \mathrm{~min}$. After each incubation step, the cells were washed three times with PBS for 5 min each. Images of randomly selected fields of view of stained neurons were captured using an Olympus IX71 microscope (Olympus Corp., Tokyo, Japan).

Morphological analysis. Cortical neurons were cultured in 24-well plates at a density of $2.0 \times 10^{4}$ cells $/ \mathrm{cm}^{2}$ and treated for three days with or without senegenin $(2 \mu \mathrm{M}), \mathrm{K} 252 \mathrm{a}(50 \mathrm{nM})$, ZM241385 (10 nM), GÖ6976 (100 nM), PD98059 (10 $\mu \mathrm{M})$ or LY294002 $(10 \mu \mathrm{M})$ as described previously (15-18). Neurons were fixed and stained with MAP2 antibody to observe the morphology of cortical neurons. Images of randomly selected fields of view of stained neurons were captured using an Olympus IX71 microscope (Olympus Corp.) at x400 magnification and the average length of neurite outgrowth in each group was measured using Image Pro Plus 6.0 software (Media Cybernetics, Rockville, MD, USA) by evaluating 200 neurons in a randomly selected field of view.

Western blot analysis. Cortical neurons were cultured in a six-well plate at a density of $2.5 \times 10^{5} \mathrm{cells} / \mathrm{cm}^{2}$ and treated with or without senegenin and $10 \mu \mathrm{M} \mathrm{LY} 294002$ for three days. Following washing with $1 \mathrm{ml}$ ice-cold PBS, cells were lysed in $100 \mu \mathrm{l}$ lysis buffer [115 mM Tris-HCl (pH 6.8), 4\% $(\mathrm{w} / \mathrm{v})$ SDS, $10 \%(\mathrm{v} / \mathrm{v})$ glycerol, $10 \mathrm{mM}$ dithiothreitol and $2.5 \mathrm{mg} / \mathrm{ml}$ bromophenol blue]. Protein was quantified using a BCA Protein Quantification kit. Equivalent amounts of protein $(10 \mu \mathrm{g})$ were subjected to $12 \%$ SDS-PAGE and transferred onto a PVDF membrane, followed by incubation of the membrane with antibodies against phospho (p)-Akt (1:2,000 dilution), Akt (1:1,000 dilution) and GAPDH (1:1,000 dilution). Subsequently, membranes were incubated with the HRP-conjugated secondary antibody (1:3,000 dilution). The non-phosphorylated form of Akt from the same cell lysates was quantified by immunoblotting to ensure that phosphorylated Akt was detected in an identical amount of protein. The blots were visualized using ECL reagent. The autoradiograms were scanned and the protein level was quantified by densitometry using an image analysis system. using a Versa Doc ${ }^{\mathrm{TM}}$ Imaging system (Model 4000; Bio-Rad Laboratories, Inc., Hercules, CA, USA) and the intensity of the protein bands were analyzed using Quantity One 1-D Analysis Software v.4.6.6 (Bio-Rad Laboratories, Inc.).

Statistical analysis. Values are expressed as the mean \pm standard error of the mean. Statistical significance among groups was determined by one-way analysis of variance with Bonferroni's post hoc test. Statistical analyses were conducted using SPSS 16.0 (SPSS, Inc., Chicago, IL, USA). P $<0.05$ was considered to indicate a statistically significant difference between values.

\section{Results}

Morphology and phenotype of cultured cortical neurons. Neonatal rat cortical neurons were cultured in serum-free DMEM/F12 supplemented with $0.4 \%$ (v/v) B27 to prevent the proliferation of gliocytes. MAP2 is a neuron-specific cytoskeletal protein that is enriched in the cell body and dendrites (19). 
A

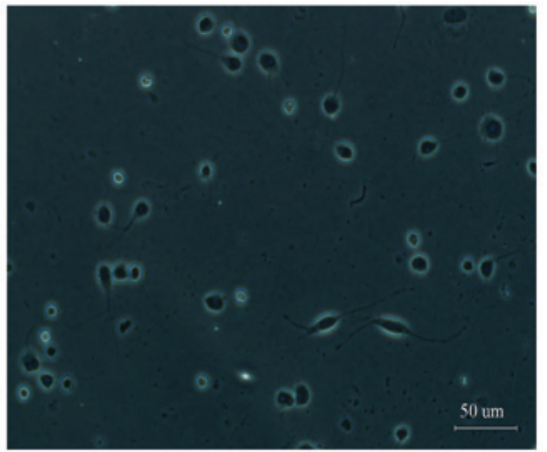

C

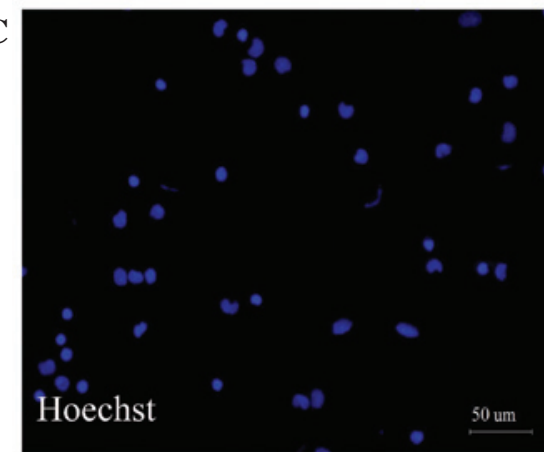

B

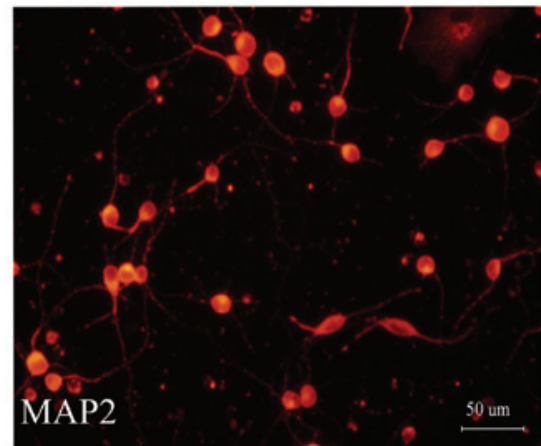

D

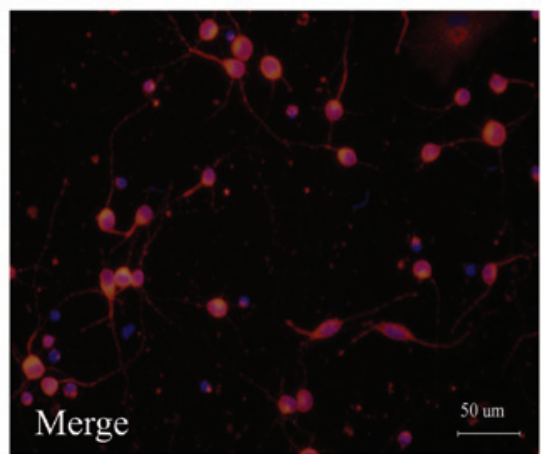

Figure 1. Identification of neuronal cells using immunofluorescence staining for MAP2. (A) Neuronal cells; (B) neuronal cells stained with MAP2 antibody; (C) neuronal cells stained with Hoechst 33258; (D) Merged image of MAP2 immunostaining and Hoechst 33258 nuclear stain in neuronal cells (scale bar, $50 \mu \mathrm{m}$ ). All cells were cultured for three days. The staining revealed that $~ 95 \%$ of the cultured cells were neurons.

A

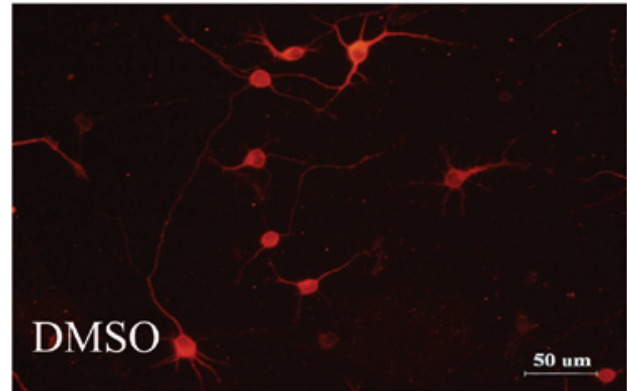

C

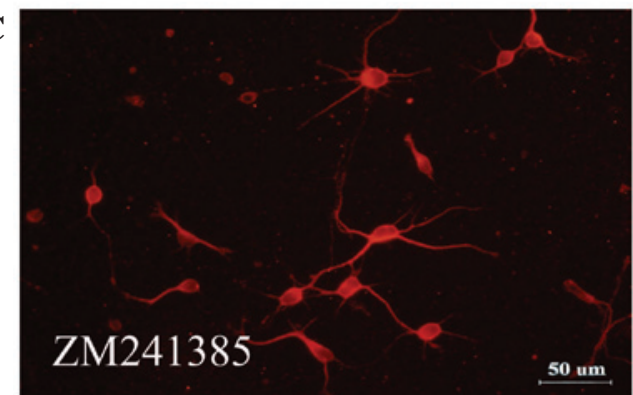

$\mathbf{E}$

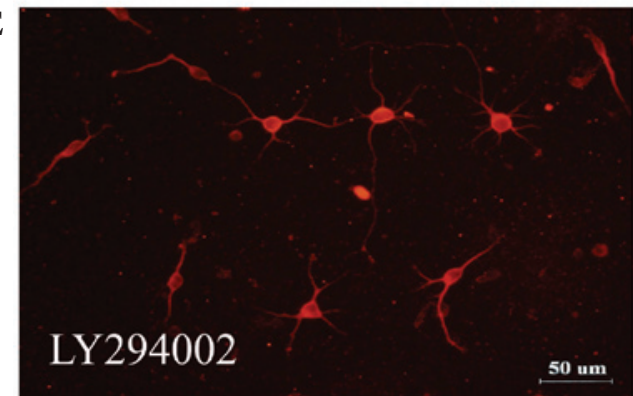

B

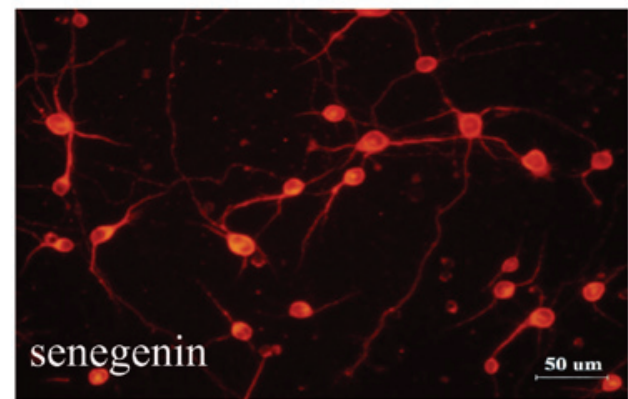

D

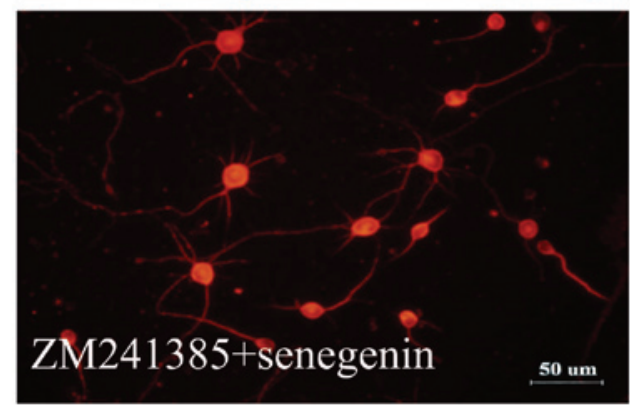

F

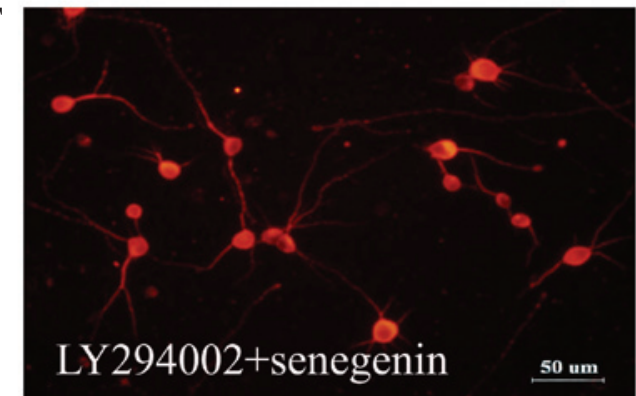

Figure 2. Effects of ZM241385 and LY294002 on senegenin-induced neurite outgrowth in cultured cortical neurons. (A) Vehicle control; (B) $2 \mu$ M senegenin;

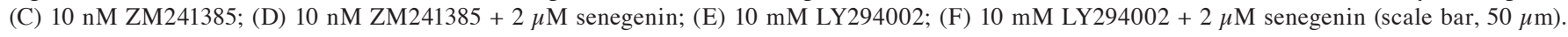
Morphological observation revealed that ZM241385 and LY294002 inhibited senegenin-induced neurite outgrowth. DMSO, dimethyl sulfoxide. 
A

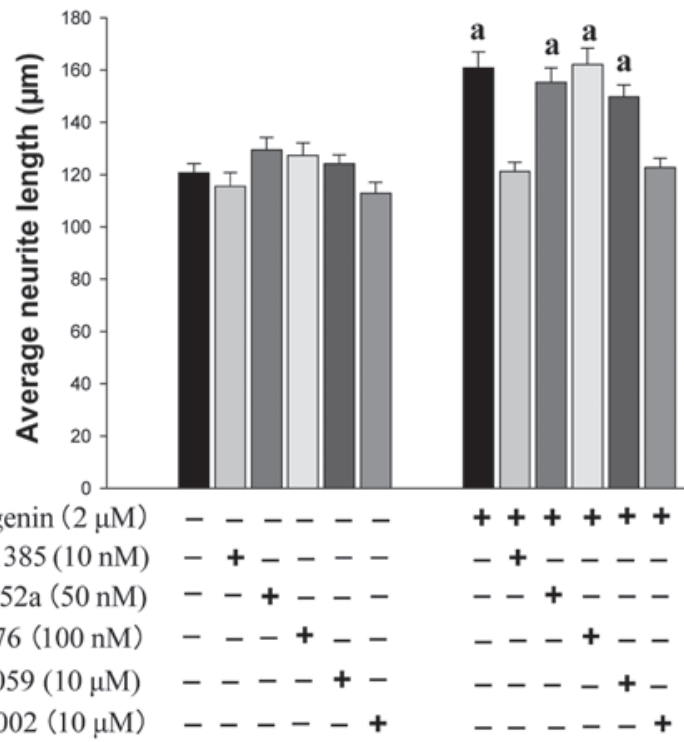

B

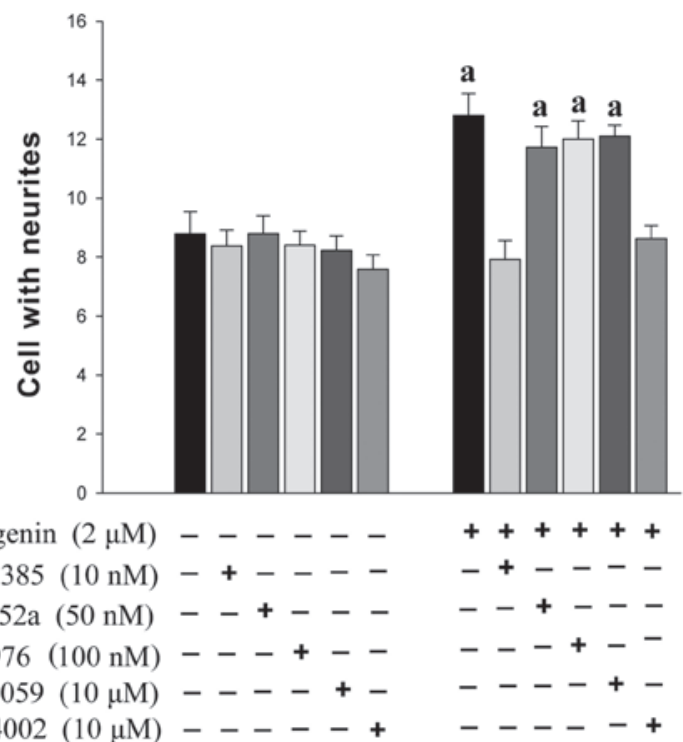

Figure 3. Neurite outgrowth and neuronal survival were measured after the neurons were treated for three days. (A) Quantitative analysis of neurite length. (B) Cells with neurites equal to or longer than the diameter of the cell body were considered to have survived and counted to analyze the effect of senegenin on the neuronal survival rate. Values are expressed as the mean \pm standard error $(n=3)$. ${ }^{a} \mathrm{P}<0.01$ compared with vehicle control.

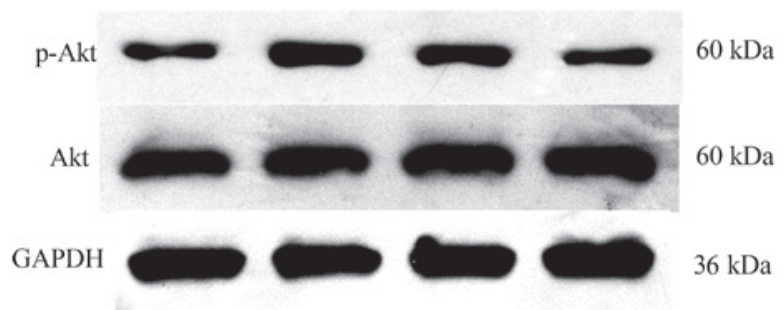

MAP2 staining can distinguish neurons from other cells. After cortical neurons were cultured for three days, MAP2 immunofluorescence staining was performed. MAP2 immunostaining revealed that $\sim 95 \%$ of the cultured cells were neurons (Fig. 1).

Senegenin promotes neurite outgrowth and neuronal survival, which is inhibited by ZM241385 and LY294002. As the phosphoinositide-3 kinase (PI3K) and the mitogen-activated protein kinase (MAPK) signaling pathways have been implicated in neurite outgrowth and neuronal survival, which is mediated via tropomyosin receptor kinase (Trk) receptors (20), the present study assessed the effect of TrkA inhibitor K252a, $\mathrm{A}_{2 \mathrm{~A}}$ receptor antagonist ZM241385, PI3K inhibitor LY294002, protein kinase C inhibitor GÖ6976 and MAPK kinase inhibitor PD98059 on senegenin-induced neurotrophic activity. The effects of senegenin on neurite outgrowth were assessed using immunostaining for MAP2. As shown in Figs. 2 and 3, the neurite outgrowth and neuronal survival induced by senegenin was prevented by treatment with ZM241385 and LY294002, but not by K252a, GÖ6976 and PD98059. These results indicated that senegenin-induced neurotrophic activity required a signaling pathway involving PI3K.

Senegenin promotes Akt phosphorylation. To further confirm the involvement of the PI3K/Akt signaling pathway in the neurotrophic effects of senegenin, the phosphorylation of Akt was investigated using western blot analysis (Fig. 4). The results demonstrated that senegenin significantly enhanced the phosphorylation of Akt as compared with that in the vehicle-treated control group. However, the enhancing effect of senegenin on Akt phosphorylation was blocked by LY294002, a specific inhibitor of PI3K, suggesting that the neurotrophic activity of senegenin may be mediated via the PI3K/Akt signaling pathway.

\section{Discussion}

NTFs and numerous neurotrophic small molecules are regulators of neuronal development, growth, survival, plasticity, 
and differentiation in the nervous system by activating Trk receptor tyrosine kinases (19-22). The results of the present study suggested that the neurotrophic effects of senegenin were counteracted with the antagonist ZM241385, an inhibitor of the $\mathrm{A}_{2 \mathrm{~A}}$ receptor, but not $\mathrm{K} 252 \mathrm{a}$, an inhibitor of Trk receptors. This indicated that the $\mathrm{A}_{2 \mathrm{~A}}$ receptor may be involved in the neurotrophic effects of senegenin, whereas previous studies (19-22) demonstrated that neurotrophic effects were mediated by receptor tyrosine kinases. $\mathrm{A}_{2 \mathrm{~A}}$ receptors are a type of $\mathrm{G}$ protein-coupled receptors, which are expressed in the neuropil of the striatum, the cortex, hippocampus, cerebellum, and olfactory striatum (23). Furthermore, the $\mathrm{A}_{2 \mathrm{~A}}$ receptor is involved in the induction of the MAPK/Erk or PI3K/Akt signaling pathway (17), which regulate neuronal differentiation, neurite outgrowth, and neuronal survival (24-26), as well as neurotrophic effects (27). In addition, Liot et al (28) demonstrated that NT-3 displayed an anti-apoptotic effect on cultured cortical neurons via activation of the PI3K/Akt signaling pathway. To date, numerous previous studies have demonstrated that the PI3K/Akt signaling pathway has an important role in mediating the survival and/or neurite outgrowth processes. Ashcroft et al (29) demonstrated that the selective and inducible activation of endogenous PI3K in PC12 cells results in efficient NGF-mediated survival and neurite outgrowth. In addition, López-Carballo et al (30) showed that the PI3K/Akt signaling pathway is important in the regulation of RA-induced neuronal survival in SH-SY5Y human neuroblastoma cells. Zhang et al (31) showed that the PI3K/Akt signaling pathway contributed to neuronal survival and neurite outgrowth induced by methyl 3,4-dihydroxybenzoate. The results of the present study demonstrated that the PI3K inhibtor LY294002, but not the MEK inhibitor PD98059, prevented senegenin-induced neurotrophic activity. Akt phosphorylation was enhanced in cortical neurons treated with senegenin but phosphorylation levels were attenuated by LY294002. Therefore, these results indicated that PI3K activation is essential for senegenin-induced neurite outgrowth and neuronal survival in cortical neurons, and also suggested that Akt phosphorylation was enhanced in cortical neurons treated with senegenin, but could be attenuated by LY294002. The data also suggested that Akt phosphorylation has a role in neurite outgrowth and survival induced by senegenin. Senegenin may enhance Akt activity by activating $\mathrm{A}_{2 \mathrm{~A}}$ receptors in order to exert its neurotrophic activity. The neurotrophic effects may be important in the protection of neurons against damage. Honokio, magnolol and methyl 3,4-dihydroxybenzoate exhibit neurotrophic effects, and these compounds are also known to protect neurons from various types of damage (32-34). Brain amyloid $\beta(\mathrm{A} \beta)$ aggregates into clumps called oligomers that can accumulate and form deposits called amyloid plaques, which are thought to be a pathological mechanism underlying Alzheimer's disease $(35,36)$. Previous studies demonstrated that $\mathrm{A} \beta$ may cause neuronal death $(34,37)$. Future studies will clarify the neuroprotective effects of senegenin against $\mathrm{A} \beta$-induced apoptosis in rat primary cortical neurons.

In conclusion, the results of the present study demonstrated that the $\mathrm{A}_{2 \mathrm{~A}} / \mathrm{PI} 3 \mathrm{~K} / \mathrm{Akt}$ signaling cascade is involved in the neurotrophic effects of senegenin. These results provide novel insights for treating Alzheimer's disease and neurodegenerative diseases. Further studies are currently taking place to clarify which downstream proteins are activated by Akt, and which molecules in neurons are associated with senegenin-induced neurotrophic activities.

\section{Acknowledgements}

This present study was supported by grants from the National Natural Science Foundation Committee of China (grant nos. 81173037, 30672450 and 81202519) and the Guangdong Provincial Department of Science and Technology (grant nos. 2012B050300018 and 2010B030700018).

\section{References}

1. Christensen DZ, Bayer TA and Wirths O: Intracellular A $\beta$ triggers neuron loss in the cholinergic system of the APP/ PS1KI mouse model of Alzheimer's disease. Neurobiol Aging 1153-1163, 2008.

2. Mullen LM, Pak KK, Chavez E, Kondo K, Brand Y and Ryan AF: Ras/p38 and PI3K/Akt but not Mek/Erk signaling mediate BDNF-induced neurite formation on neonatal cochlear spiral ganglion explants. Brain Res 1430: 25-34, 2012.

3. Zhai Hf, Nakatsukasa M, Mitsumoto Y and Fukuyama Y: Neurotrophic effects of talaumidin, a neolignan from Aristolochia arcuata, in primary cultured rat cortical neurons. Planta Med 70: 598-602, 2004.

4. Pardridge WM. Neurotrophins, neuroprotection and the blood-brain barrier. Curr Opin Investig Drugs 3: 1753-1757, 2002.

5. Lee YK, Choi IS, Kim YH, Kim KH, Nam SY, Yun YW, Lee MS, Oh KW and Hong JT: Neurite outgrowth effect of 4-O-methylhonokiol by induction of neurotrophic factors through ERK activation. Neurochem Res 34: 2251-2260, 2009.

6. Lee MM, Hseih MT, Kuo JS, Yeh FT and Huang HM: Magnolol protects cortical neuronal cells from chemical hypoxia in rats. Neuroreport 9: 3451-3456, 1998.

7. Chang CP, Hsu YC and Lin MT: Magnolol protects against cerebral ischaemic injury of rat heatstroke. Clin Exp Pharmacol Physiol 30: 387-392, 2003.

8. Moriyama M, Huang JM, Yang CS, Kubo M, Harada K, Hioki $\mathrm{H}$ and Fukuyama $\mathrm{Y}$ : Two new sesquiterpenoids and two new prenylated phenylpropanoids from Illicium fargesii and neuroprotective activity of macranthol. Chem Pharm Bull (Tokyo) 56: 1201-1204, 2008.

9. Jia HX, Jiang Y, Ruan Y, Zhang Y, Ma X, Zhang J, Beyreuther K, $\mathrm{Tu} \mathrm{P}$ and Zhang D: Tenuigenin treatment decreases secretion of the Alzheimer's disease amyloid beta-protein in cultured cells. Neuroscience Lett 367: 123-128, 2004.

10. Sun GB, Deng XC and Li CH: The protective effects of tenuigenin on the PC12 cells injury induced by $\mathrm{H} 2 \mathrm{O} 2$. Journal of Chinese Medicinal Materials 30: 991-993, 2007 (In Chinese).

11. Park C, Choi SH, Koo JW, Seo JH, Kim HS, Jeong SJ and Suh YH: Novel cognitive improving and neuroprotective activities of Polygala tenuifolia Willdenow extract, BT-11. J Neurosci Res 70: 484-492, 2002.

12. Pi T, Xue XY, Lin LF and Luo HM: Neurotrophic effects of senegenin on newborn rat cultured cortical neurons. Chin J Pharmacol Toxicol 25: 40-44, 2011.

13. Pi T, Xue XY and Luo HM: Neurotrophic effects of senegenin. Journal of Chinese Medicinal Materials 9: 1477-1480, 2013 (In Chinese).

14. Lin LF, Xue XY, Liao MJ, Xiao F, Lv RH and Luo HM: Neurotrophic effects of magnesium fructose 1,6-diphosphate on cortical neurons. Int J Neurosci 122: 248-254, 2012.

15. Matsuda S, Saito H and Nishiyama N: Effect of basic fibroblast growth-factor on neuron cultured from various regions of postnatal rat-brain. Brain Res 520: 310-316, 1990.

16. Tassi E, Walter S, Aigner A, Cabal-Manzano RH, Ray R, Reier PJ and Wellstein A: Effects on neurite outgrowth and cell survival of a secreted fibroblast growth factor binding protein upregulated during spinal cord injury. Am J Physiol Regul Integr Comp Physiol 293: R775-R783, 2007.

17. Lee FS and Chao MV: Activation of Trk neurotrophin receptors in the absence of neurotrophins. Proc Natl Acad Sci USA 98: 3555-3560, 2001. 
18. Xiao F, Lin LF, Cheng X, Gao Q and Luo HM: Nogo-66 receptor activation inhibits neurite outgrowth and increases $\beta$-amyloid protein secretion of cortical neurons. Mol Med Rep 5: 619-624, 2012.

19. Serres and Carney SL: Nicotine regulates SH-SY5Y neuroblastoma cell proliferation through the release of brain-derived neurotrophic factor. Brain Res 1101: 36-42, 2006.

20. Orike N, Thrasivoulou C, Wrigley A and Cowen T: Differential regulation of survival and growth in adult sympathetic neurons: An in vitro study of neurotrophin responsiveness. J Neurobiol 47: 295-305, 2001.

21. Rajagopal R, Chen ZY, Lee FS and Chao MV: Transactivation of Trk neurotrophin receptors by G-protein-coupled receptor ligands occurs on intracellular membranes. J Neurosci 24 6650-6658, 2004.

22. Lee FS and Chao MV: Activation of Trk neurotrophin receptors in the absence of neurotrophins. Proc Natl Acad Sci USA 98: 3555-3560, 2001

23. Rosin DL, Robeva A, Woodard RL, Guyenet PG and Linden J: Immunohistochemical localization of adenosine A2A receptors in the rat central nervous system. J Comp Neuro 401: 163-186, 1998.

24. Kaplan DR and Miller FD: Neurotrophin signal transduction in the nervous system. Curr Opin Neurobiol 10: 381-391, 2000.

25. Ditlevsen DK, Køhler LB, Pedersen MV, Risell M, Kolkova K, Meyer M, Berezin V and Bock E: The role of phosphatidylinositol 3-kinase in neural cell adhesion molecule-mediated neuronal differentiation and survival. J Neurochem 84: 546-556, 2003.

26. López-Carballo G, Moreno L, Masiá S, Pérez P and Barettino D: Activation of the phosphatidylinositol 3-kinase/Akt signaling pathway by retinoic acid is required for neural differentiation of SH-SY5Y human neuroblastoma cells. J Biol Chem 277: 25297-25304, 2002.

27. Ohta H, Arai S, Akita K, Ohta T and Fukuda S: Neurotrophic effects of a cyanine dye via the PI3K-Akt pathway: Attenuation of motor discoordination and neurodegeneration in an ataxic animal model. Plos One 6: e17137, 2011.

28. Liot G, Gabriel C, Cacquevel M, Ali C, MacKenzie ET, Buisson A and Vivien D: Neurotrophin-3-induced PI-3 kinase/Akt signaling rescues cortical neurons from apoptosis. Exp Neurol 187: 38-46, 2004 .
29. Ashcroft M, Stephens RM, Hallberg B, Downward J and Kaplan DR: The selective and inducible activation of endogenous PI3-kinase in PC12 cells results in efficient NGF-mediated survival but defective neurite outgrowth. Oncogene 18: 4586-4597, 1999.

30. López-Carballo G, Moreno L, Masiá S, Pérez P and Barettino D: Activation of the phosphatidylinositol 3-kinase/Akt signaling pathway by retinoic acid Is required for neural differentiation of SH-SY5Y human neuroblastoma cells. J Biol Chem 277: 25297-25304, 2002.

31. Zhang Z, Cai L, Zhou X, Su C, Xiao F, Gao Q and Luo HM: Methyl 3,4-dihydroxybenzoate promote rat cortical neurons survival and neurite outgrowth through the adenosine A2a receptor/PI3K/Akt signaling pathway. Neuroreport 26: 367-373, 2015.

32. Chang CP, Hsu YC and Lin MT: Magnolol protects against cerebral ischaemic injury of rat heatstroke. Clin Exp Pharmacol Physiol 30: 387-392, 2003.

33. Liou KT, Shen YC, Chen CF, Tsao CM and Tsai SK: Honokiol protects rat brain from focal cerebral ischemia-reperfusion injury by inhibiting neutrophil infiltration and reactive oxygen species production. Brain Res 992: 159-166, 2003.

34. Zhou XW, Zhang Z,Su CF,LvRH,Zhou X, Cai L, Wang CY, Yan L, Zhang W and Luo HM: Methyl 3,4-dihydroxybenzoate protects primary cortical neurons against $\mathrm{A} \beta_{25-35}$-induced neurotoxicity through mitochondria pathway. J Neurosci Res 91: 1215-1225, 2013.

35. Chui DH, Dobo E, Makifuchi T, Akiyama H, Kawakatsu S, Petit A, Checler F, Araki W, Takahashi K and Tabira T: Apoptotic neurons in Alzheimer's disease frequently show intracellular Abeta42 labeling. J Alzheimers Dis 3: 231-239, 2001.

36. Qian $\mathrm{YH}, \mathrm{Xiao} \mathrm{Q}$ and $\mathrm{Xu} \mathrm{J}$ : The protective effects of tanshinone IIA on $\beta$-amyloid protein (1-42)-induced cytotoxicity via activation of the Bcl-xL pathway in neuron. Brain Res Bull 88: 354-358, 2012.

37. Christensen DZ, Bayer TA and Wirths O: Intracellular A $\beta$ triggers neuron loss in the cholinergic system of the APP/PS1KI mouse model of Alzheimer's disease. Neurobiol Aging 31: $1153-1163,2010$ 